the intestines are all matted together, so that you can take them out as a whole, and when you cut across the mass, the intestines are held open by the thickening of the peritoneum around them, the mesentery shrinks and draws the intestines back, and the omentum gets puckered up into a great band which lies transversely across the colon. In these cases usually there is universal thickening of the capsule of the spleen as well, and in many of them granular kidneys are present, so that it must sometimes be regarded as a complication of granular kidney; sometimes there is an alcoholic history, and occasionally the patients have had syphilis.

You will say, "What is the misconception in regard to that?" It is this, there is a tendency to lump together the partial perihepatitis and the general. The partial is unimportant; in it there are little patches here and there; it is not part and parcel of a general peritonitis. But the universal perihepatitis, with thick white capsule over the liver is a much more important condition, for it is part of a general chronic peritonitis. For want of time I have had to confine myself to the peritoneum, but sometimes a zuckergussleber is associated not only with chronic peritonitis but with chronic pericarditis and chronic pleurisy. To this condition the term multiple progressive hyaloserositis has been applied by Nicholls. ${ }^{1}$ He, I may add, quite supports my contention that general perihepatitis is usually merely part of a chronic peritonitis.

Primary Malignant Dishase of the Liver.

There is a wide divergence of opinion as to the frequency of primary malignant disease of the liver. Friedreichs gives the proportion of primary to secondary malignant disease of the liver as one to two. On the other hand, you come across people who doubt whether there is such a thing as primary malignant disease of the liver. I went through our necropsy records. Among 10, ooo necropsies we had ro cases of undoubted primary malignant disease of the liver. That is to say every part of the body was carefully searched, but no other part of the body was found to contain malignant disease at all, except that sometimes the portal glands were a little affected by secondary deposits; and sometimes the few glands there are by the diaphragm on the upper surface of the liver. To show the care with which the cases were examined, I may say that in one case in which the patient had moles on the skin every mole on the body was subjected to a microscopical examination, to make sure that it was not the primary seat of the disease, because the growth was melanotic. The figures worked out in this way. Taking 10,000 necropsies, we had I case of undoubted primary malignant disease of the liver to every 24 in which the malignant disease was secondary to growth elsewhere. If we take the cases in which there could be no real doubt that the malignant disease in the liver was primary, but in which a few deposits aecondary to the malignant disease of the liver had occurred in other parts of the body, it works out as I case of primary malignant disease in the liver to 17 in which the disease of the liver is secondary to malignant disease elsewhere. These figures, therefore, wtrike the balance between believing that it rarely, if ever, occurs, and the very high estimate given by Friedreichs of I to 2 . I suspect that one cause at least of the mistake is that it has been overlooked that primary disease can occur in the gall bladder and biliary passages, and that malignant disease of the liver may be secondary to primary disease in those parts. While we are on the subject, I might point out a few clinical symptoms of primary malignant disease of the liver. It usually occurs at the same age as other malignant disease. In 17 cases, 10 were men and 7 women. After the first $8 y m$ ptom was observed in those in which the date was sufticient to draw deductions, 2 lived four months, 2 lived three months, 1 lived two and a-half months, 2 lived two months. Therefore it is a disease very rapidly fatal, much more so than secondary malignant disease of the liver. Pain and tenderness were usual, and unless the primary malignant disease was of that very rare variety, scirrhus of the liver which contracts the organ, the liver was always enlarged, and weighed usually between 120 and $130 \mathrm{oz}$; once the enormous weight of $267 \mathrm{oz}$. was attained. Now this is important; out of ro cases there was no jaundice in four, and in two jaundice did not appear until just before death; in three of those affected it was slight. That is a very striking contrast to secondary malignant disease of the liver, in which iaundice is such a prominent feature. Never in primary malignant disease of the liver did jaundice become deep olive green, probably because the patient did not live long enough; and the absence of the jaundice could not be entirely attributed to the fact that there was not time for the glands in the transverse fissure to. be enlarged secondarily to the disease in the liver, because sometimes they were enlarged and no jaundice was present. Ascites was present in about half the cases. The temperature was often raised. We know it is raised occasionally in cirrhosis of the liver and in cancer of the liver, but it appeared to be particularly raised when the disease was primary in the liver. So $I$ suggest that in attempting to diagnose whether malignant disease of the liver is primary or secondary we should pay attention to the following points: Is. the disease very rapid? You see no patient lived more than four months after it was recognized. If it is rapid that fact is an argument in favour of the disease in the liver being primary. In the next place, if there is no jaundice it is an argument in favour of the disease being primary in the liver, and so perhaps also is an unusually high temperature.

Since I wrote the above many of the points urged have been confirmed by a paper by Acland and Dudgeon. ${ }^{2}$

Local Peritonitis dof to Gall Stones.

The only other point to which I have time to direct attention is a condition which I do not think we sufficiently often bear in mind in thinking of liver disease, and that is one of the after-effects of gall stones. It is not by any means an uncommon thing at a necropsy to find a good deal of local peritonitis around the gall bladder, and that may well cause the stomach and duodenum to become adherent to the gall bladder, and then the patient will have all sorts of vague pains in the abaomen, sometimes very severe, sometimes making life a misery and causing her to be called a hypochondriac and a valetudinarian. If we go carefully into the history, and find there is a distinct history of gall stones it may help us to come to a diagnosis. Perhaps I can best illustrate this point by two cases. In one case which I saw there was dilatation of the stomach, and inasmuch as a mass could be felt, it was thought that the dilatation was due to growth. Post mortem I found that the patient had much matting around the gall bladder, in which there were some stones, and that the gall bladder was adherent to the duodenum, and that all this matting around the first part of the duodenum and the pylorus had led to constriction at the pylorus and the consequent dilatation of the stomach, and all the symptoms of which the patient complained. In the next case, an old woman complained of great pain in the abdomen, which was much worse after food. She complained that she had indigestion, and sometimes she rolled about in pain. She gave a history of gall stones. The diagnosis made was that there were adhesions about the gall bladder implicating the end of the stomach and the duodenum. The abdomen was opened and the diagnosis was found to be correct. The adhesions were freed and the patient's pain was relieved.

1 Studies from the Royal Victoria Hospital, Montreal, vol. i, No. 3. ${ }^{2}$ Luncet November 15 th, 1902

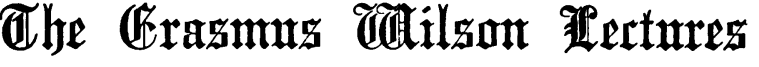

\section{ADENOMA AND ADENO-CARCINOMA OF THE RECTUM.}

Delivered before the Royal College of Surgeons of England.

By Sir CHARLES B. BALL, M.CH., F.R.C.S.I., HON.F.R.C.S.ENG.

Regius Professor of Surgery in the University of Dublin.

LeCTURE III.

Surgical Treatment of Cancer of the Rectum. HAVING determined that a given case of cancer of the rectum is suitable for removal, that is, that it is freely movable or at any rate only slightly invades the perirectal tissues; it is necessary to consider in the first place what route is the best adapted to the individual case, and this will largely depend upon the situation of the disease : (1) the growth may involve the anal canal only; (2) it may be situated low down in the rectal ampulla ; and (3) it may originate high up above the peritoneal reflection; it must, however, be remembered that the disease may be so extensive as to occupy situations 1 and 2 or 3 and 4 , while in some rare cases the entire rectum including the anal canal may be implicated. The routes we 
have to select from are: (1) the perineal; (2) the sacral; (3) the vaginal; and (4) the abdominal.

Preparation of Patient.

Before undertaking any of these procedures, a thorough and intelligent preliminary preparation of the patient is probably of greater importance than in alrnost any other surgical operation ; four or five days before free purgation should be commenced, and continued till the day previous to operation, when a copious enema should suffice to completely empty the colon, the patient is to be well fed with food that will leave but little residue, and the night before operation a full opiate is to be administered to check peristalsis. Another enema may be given a couple of hours before operation, and when the patient is well under ether, a thorough washing-out of the rectim and lower part of the colon with a 2 per cent. solution of creolin or other antiseptic solution should be employed; this preliminary preparation is a matter of very real importance. It is well known that the septicity of the intestinal contents increases the longer they are retained, and that a thorough evacuation, carried out during several days previously, tends largely to diminish the risk of wound contamination in these cases; but it may not be possible to effect this thorough evacuation, large masses of scybala may block the colon above the seat of disease or the stricture may be so tight that complete obstruction has supervened, these are cases in which

\section{A Previous Colotomy}

is clearly indicated; some surgeons, indeed, recommend colotomy previous to all excisions of the rectum, but where the bowel can be efficiently evacuated, I am satisfied that it is unnecessary and objectionable, as it causes delay and subjects the patient to a double operation; where, however, complete evacuation is impossible without colotomy, it should be certainly undertaken as a preliminary, or if obstruction is complete and attended with much meteorism, the method of intestinal drainage, introduced by the late Mr. Greig Smith, is probably preferable.

Perineal Excision,

formerly the only method employed for the removal of rectal cancer, is now not so frequently undertaken, and should, I believe, only be adopted in cases which involve the anal canal, as incontinence of faeces is a necessary result of excision of cancer in this way; when, however, the anal canal is implicated, the incisions necessary for complete removal must so involve the musculature that incontinence will certainly follow. If only portion of the circumference of the bowel is infiltrated, an incision is to be carried round that portion of the anus corresponding with the disease, and the levator ani divided to a similar extent. Vertical incisions are then to be made on either side, but well free of the growth, and carried up the rectum to a point gufficiently above the cancer, the flap thus formed is to be freed from its connexions, drawn down, and a clamp applied to the healthy tissue above; the disease is then cut away, any vessels bleeding are to be ligatured and the cut end of mucous membrane attached to the skin by deep and superficial sutures. In passing the deep sutures care must be taken that they are passed not only through skin and rectal wall but also under all the perirectal tissues and in sufficient number to obliterate any cavity outside the rectum in which blood or serum may collect; this is a matter of high importance in the prevention of sepsis. The contrast between wounds of the anal canal alone, such as pile and many fistula operations, and those wounds whieh penetrate the entire rectal tunic and so snen up the superior pelvirectal space is very marked, sepsis to any serious extent being excessively rare after pile operations, but common after wound such as excision of the rectum which open through the rectal wall above the pelvic diaphragm, yet both these wounds are exposed to the same sources of infection from intestinal contents ; there can, 1 think, be no doubt that the difference is due to the fact that once the superior pelvirectal space is opened up, blood and serum are likely to be widely diffused and form a suitabie culture medium for the growth of bacteria; it is, therefore, imperative to close the deeper portion of the wound efficiently by deep sutures, or if this is found to be impracticable; to ensure free drainage by means of tubes or iodoform gauze packing.

When the disease extends round the entire circumference of the anal canal, excision can be carried out in like manner; the amputated rectum being sutured to the skin.

In connexion with perineal excision, it is well to remember that, in some rare instances, it may be possible to prolapse the entire disease through the anus. This may be the case in single nodules occupying portion of the circumference of the bowel low down, and also when the disease completely surrounds the intestine, either rectum or colon, an intussusception may be induced which becomes long enough for the cancer to reach the anus; when it is possible thus to extrude the entire growth through the anus, excision can be very easily accomplished, the wound carefully sutured being returned within the anus; such cases usually do well, as the fact that the disease can be protruded indicates that the perirectal tissues are not as yet involved.

\section{SaCral Route.}

In the early days of perineal excision it soon became evident that this route was unsuitable for cancers which extended a considerable distance up the rectum; it was therefore sought to obtain more room, first by posterior incision. then the coccyx was removed, and finally, by Kraske's sacral incision, a great impetus was given to the efficient treatment of extensive cases. 'Kraske's original incision has been much modified by other surgeons; the method which appears to be best is as follows:-The skin over the sacrum is to be cleansed antiseptically, and an incision made from the middle of the sacrum to within about an inch of the anus (Fig. 1); this

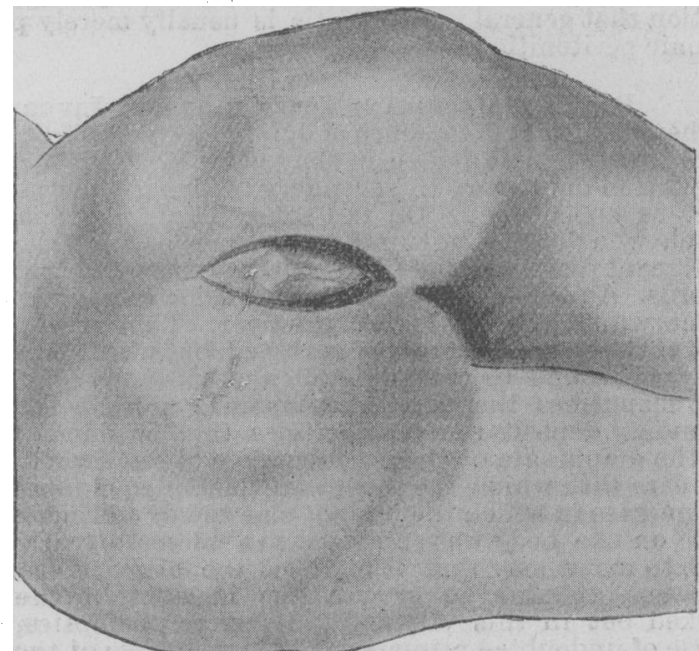

Fig. x.-First incision in trans-sacral excision of the rectum, exposing the coccyx and portion of the sacrum, with their muscular and ligamentous connexions.

exposes the fibrous coverings of the bone with the attached ligaments, and the connexions of the levatores ani to the coccyx, and to one another in the middle line. An incision is now made through the musculature below the tip of the coccyx, and with American pruning shears the ljgaments, first on one side of the coccy $x$ and then on the other,

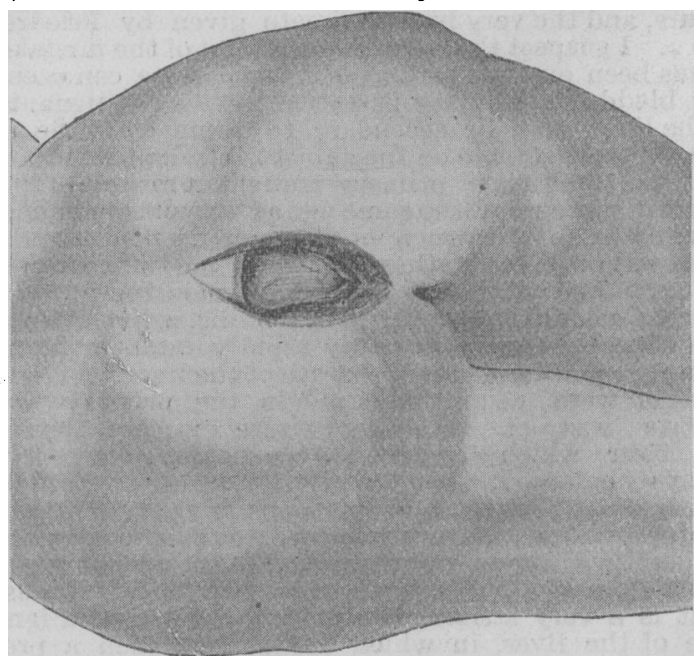
Fig. 2.-Removal of coccyx and last bone of the -sacrum, exposing I 
are severed, while a third section is carried transversely through the fifth sacral vertebra, and the triangle of bone thus separated from its attachments is easily removed. This transverse division of the sacrum is practically that recommended by Bardenheuer, which 1 consider far the best line of section (Fig. 2). When made through the fifth sacral vertebra, sufficient room is usually secured, but a further bone section can readily be made if required; it is, however, of great importance to keep it as low as possible, so as to ensure the safety of the nervous supply of the sphincteric apparatus. The removal of the entire piece of bone is unattended with subsequent trouble, and is, I think, vastly to be preferred to any of the osteoplastic operations which have been recommended. The American pruning shears cut the bone cleanly without much bruising, and much more rapidly than any other instrument.

The posterior surface of the rectum is now exposed and separated with care, bleeding of course being arrested, but usually only the mid-sacral artery requires attention. With the finger now an attempt should be made to free the anterior surface so as to clear the entire circumference of the rectum; in the female this can sometimes be done with ease, but it is usually difficult in the male. No force or bruising is permissible, so, if difliculty arises, the bowel should be divided circumferentially before the anterior connexions are separated. When the disease is tolerably low down, this incision is best made above; when high up, below; while occasionally in cases of very severe disease it may be wiser to make the incision through the infiltrated intestinal wall, subsequently removing the diseased structure from each end. This incision should be made with a pair of scissors bit by bit, pressure forceps being applied to the bleeding points, irrigation is to be carried out during the whole procedure. As soon as the entire circumference is freed, the upper lumen of the bowel is closed by a suitable clamp, and we now have to decide whether the case is one in which a sacral anus should be made; an end-to-end resection be performed; or the lower portion completely removed without injuring the pelvic diaphragm or sphincteric apparatus, and the upper portion brought out and fixed at the normal anus. Formerly a very large proportion of cases were completed by the formation of a sacral anus; and in my own series a large number of the earlier cases were thus terminated, although I now know that many of them were suitable for an operation that would retain sphincteric control with an anus normally situated. I believe that a sacral anus is necessary only in a very small proportion of cases-namely, those in which the disease is extremely extensive, and involves the anus to such an extent that it is impossible to retain the surrounding musculature in any useful condition, and also in those rare cases in which it is found impossible to free the upper segment sufficiently to bring it down for a resection or normally-situated artificial anus. Such cases are, however, exceptional. Should, however, the case be considered one in which a sacral anus is necessary, the upper segment is to be freed above the disease which is to be removed making sure that the section traverses the bowel well free of infiltration. The free lumen is now to be brought out over the divided sacrum and sutured to the skin and the large cavity left by the removal completely closed by deep and superficial sutures.

A resection with end-to-end junction, if a fair amount of healthy bowel exists below the disease, may sometimes be a possibility, and in the rare case in which it succeeds the result is very perfect, the conditions under which this operation are performed, however, being extremely unfavourable for good union. In the first place, there is not a uniform covering of peritoneum; secondly, the solid faeces of the colon exercise a considerable tension upon the bond of union. while to dilate the anus for the passage of faeces, even under normal conditions, necessitates a, very considerable strain, and if, as is usually the case, the bowels are kept confined for many days after operation, this strain will be so great as almost certainly to tear through the new bond of union even if tolerably firm. It is well, therefore, if the surgeon attempts this method, to commence free saline purgation after the second day, and in this way as far as practicable to assimilate the case to that of the small intestine with its fluid contents, where we know the results of end-to-end junction are so much better than they are in the colon. The method of junction may be by suture, decalcified bone ring, or Murphy's button, at the discretion of the surgeon.

In three cases in which I attempted to get end-toend junction, a posterior fistula formed, and although a good result was finally obtained after closure of the fistula $I$ inow think it better to suttare only the front and sides of the bowel in the first instance, leaving the posterior portion open, and close this posterior opening by a subsequent plastic operation. In a case in which $I$ adopted this method the line of union was more satisfactory.

The method which promises the best results in a very large majority of cases is undoubtedly that of Moulonguet. 1 He dissects out the lower portion to the upper surface of the pelvic diaphragm, and the entire mucous membrane down

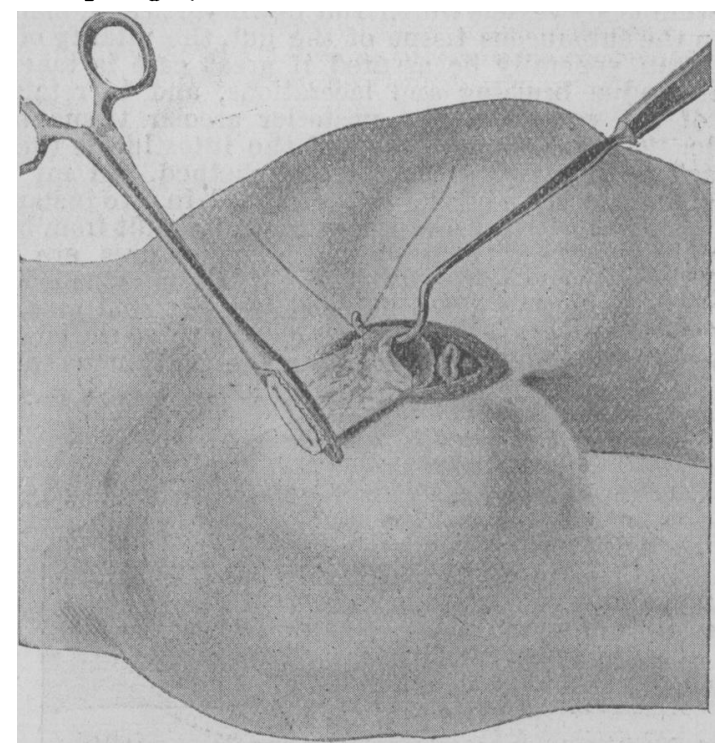

Fig. 3. - The way to pass sutures so as to tie off the attachments of the upper portion of the rectum before dividing them with to its junction with the scaly epithelium of the anal canal, but carefully preserves the entire sphincteric apparatus from injury, and then frees the upper end sufficiently to bring it out at the anus, without tension, and suture it there, thus making an artificial anus at the normal position, completely provided with an efficient sphincteric control. There is usually no great difficulty in freeing the upper portion sufficiently to bring it down to the anus, even where an extensive excision has taken place, and in doing so it is well to open the peritoneal pouch early, as the sub sequent steps are thus much simplified, the lateral reflections being tied off and divided with scissors in the same way that broad ligaments are treated in vaginal hysterectomy (Fig. 3).

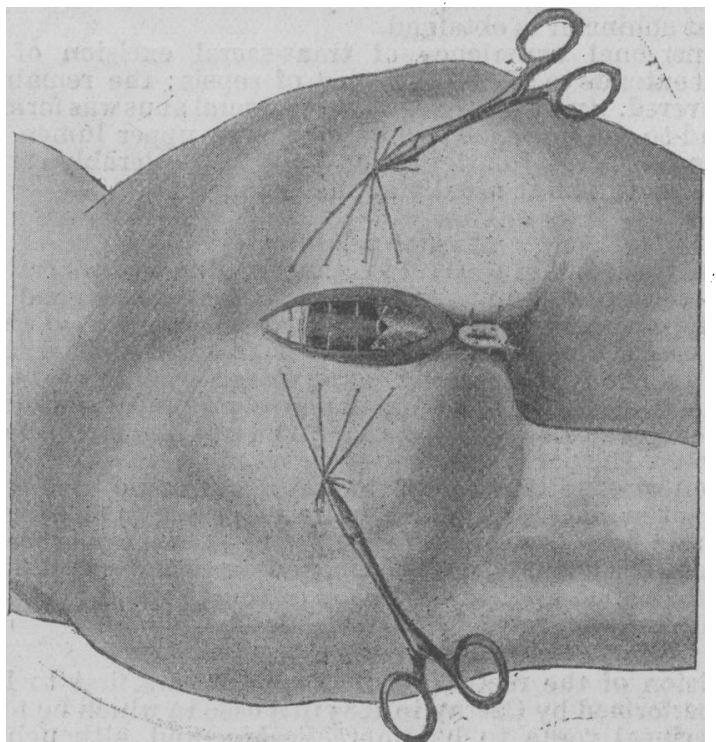

Fig. 4.-The deep sutures placed in position. The upper one passes portions of the muscular coat of the bowel, and the lower one passes through the divided attachments of the levator ani sutured to the anus. 
The abdominal cavity can be subsequently efficiently closed by uniting the free anterior edge of peritoneum by fine continuous catgut suture to the serous covering of the rectum. Posteriorly it is desirable to keep as far from the muscular tunic of the bowel as possible, remembering that the mass of vessels for the supply of the rectum pass down in the locse areolar tissue in the hollow of the sacrum, and must as far as possible be preserved. As, however, the vascular supply of the rectum is by vessels which run down vertically, many of them in the submucous tissue of the gut, the vitality of the rectum can generally be assured if great care is taken to prevent undue bruising and lacerations, and to retain as many of the vessels in the posterior areolar tissue as is possible. The danger of necrosis of the intestine is the one drawback to the employment of this method. In my own cases, a small portion of the gut sloughed in two instances, but not sufficiently to prevent an excellent result from being eventually obtained. Four or five deep sutures are now passed through the entire thickness of tissues divided, the centre ones including a few fibres of the external muscular coat of the rectum (Fig. 4). Before closing those the edges of the wound are brought together by a fine conti suous suture, and the deep sutures finally closed over lead plates (Fig. 5), no

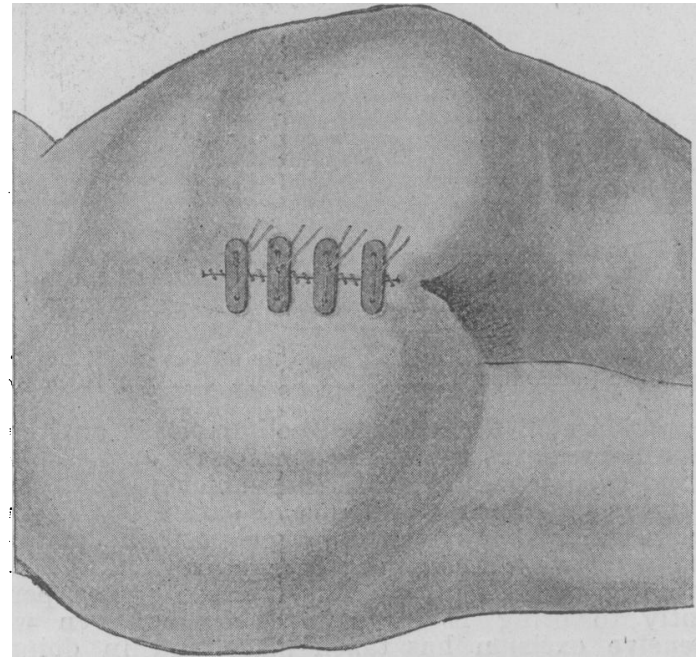

Fig. 5-The wound tinally closed, the superficial parts by continuo 'ds sutures, the deep sutures tied over lead plates.

drainage being employed. By this operation all the advantages, without the dangers, of end-to-end junction are secured, and an opening directly external controlled by an efficient sphincter is obtained.

My personal experience of trans-sacral excision of the rectum extends to 26 cases; 2 died of sepsis; the remaining 24 recovered. In 9 (mostly early cases) sacral anus was formed; in 5 end-to-end junction; while in 12 the upper lumen was fixed to the normal outlet which I now feel tolerably certain is the best and most usually applicable method.

VAGINAL RoUte.

As involvement of the recto-vaginal septum occurs eary in many cases, excision of portion of the posterior vaginal wall with the cancer has been tolerably frequently practised and in three cases under my own care this was done without difficulty, the vaginal wound being closed by sutures, but in my cases, although the operation was primarily successful, recurrence was rapid, and such must very frequently be the case where the perirectal tissues are so much invaded. The excision of cancers confined to the rectal tunic by incision through the healthy vagina appears to present a better prospect, and has been put in practice by many surgeons, especially by Murphy, of Chicago, and in suitable cases it is no doubt a good method, but I am unable to speak of it from personal experience.

AbDominal Route.

Excision of the rectum from above appears first to have been performed by Czerny in 1883 in a case in which he found the perineal route to be impracticable, and although its claims have been strongly advocated by Quénu and Hartmann, it has not yet sssumed the position in surgery which I believe it will eventually hold. The advantages are (I) after the abdomen is open it is possible to estimate exactly the xtent of disease and the degree, if any; of Jymphatic and peritoneal involvement, so that if the disease is found to be too extensive the operation can be terminated as an ordinary colotomy, or if this is considered to be unnecessary, the abdominal wound may be closed and the case allowed to run its course; (2) as the meso.colon is divided the superior haemorrhoidal artery is secured, and also the two middle baemorrhoidal arteries at an early stage, so that the operation is almost bloodless; (3) a complete removal of the lymph glands and ducts most likely to be involved is quite possible; (4) as the bowel is securely tied above and possibly below the disease, wound soiling from intestinal contents is reduced to a minimum.

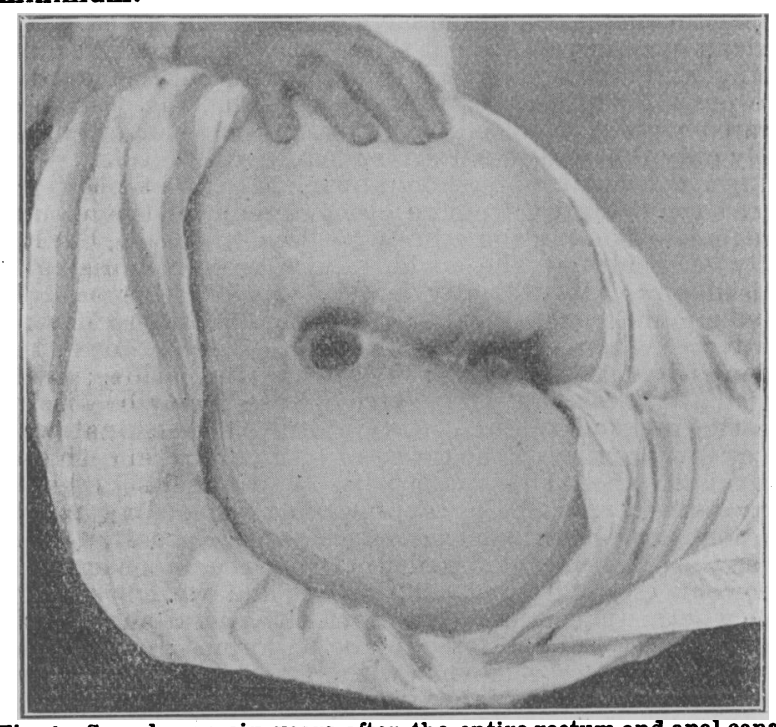

Fig. 6.-Sacral anus six years after the entire rectum and anal can had been removed by trans-sacral excision for adeno-carcinoma.

There are very important advantages which appear to me sufficient to extend largely the scope of the operation; many cases which have been treated by trans-sacral incision would I believe, have given a better result by this method, which has hitherto only been adopted in very extensive cancers of the rectum, and usually terminated by bringing out the divided colon either at the original abdominal wound or through a separate incision in the iliac region. If, of course, it is necessary to terminate the case by a permanent colotomy, this must be considered a very serious objection, but I am convinced that is possible to bring the divided colon down to and fix it in the anus in a large proportion of cases, without any wound whatever of the anal canal or pelvic diaphragm, and 80 obtain a perfect sphinteric control. The operation is best conducted as follows:-The preliminary preparation having been carried out as previously indicated, the patient is placed in the Trendelenburg position, as nearly vertical as

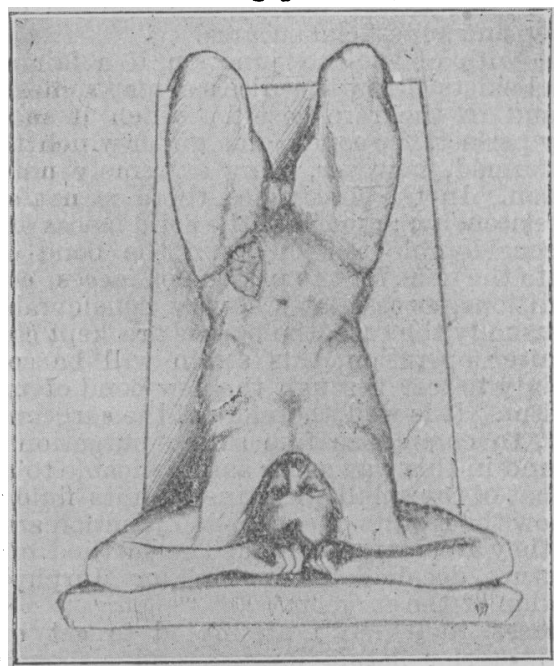

Fig. 7.-Abdominal incision of cancer of the rectum ; patient in high Trendelenburg position; the summit of the loop of pelvic colos 18 drawn out at the wound and ligatured in two pla:es. 
possible (Fig. 7), in order that the intestines may fall out of the pelvis, and so give plenty of room; the abdomen is opened by a long vertical incision, which separates the fibres of the left rectus muscle and the extent of disease determined, if movable, and the peritoneum free from infection, excision may be proceeded with, a loop of pelvic colon is drawn out and the point at which the meso-colon is longest determined, the average greatest length is about six inches, and if found as long as this the divided colon can be brought to the anus without undue tension. If, however, it proves to be considerably shorter than this limit, the case will probably have to be terminated in colotomy, the colon is ligatured in two places firmly, and divided, the cut ends being tied up in aseptic gauze, it will be observed that the point selected for division has no direct reference to the seat of disease, it is chosen so as to give as long a meso-colon as possible. The meso-colon is now divided between clamps down to its attachment (Fig. 8), and if the Paquelin cautery is

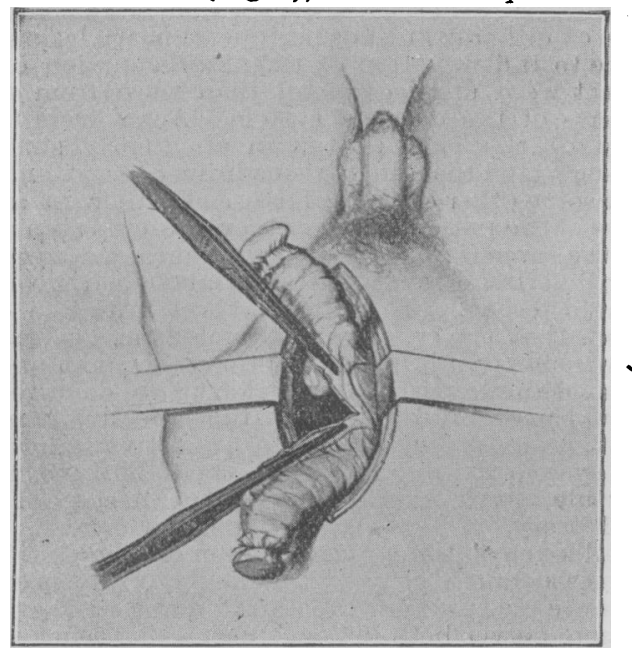

Fig. 8.-Abdominal exsision of cancer of the rectum; the colon has been cut across, and the ends covered by sterilized gavze; the meso-colon has been divided between clampyforceps duwn to its attachment by means of the Paquelin cautery.

used, top sewing of the divided edge is rendered unnecessary, the attachments of the lower portion are ligatured piece by piece, and divided until a point is reached at which the posterior surface of the rectum ceases to be covered by peritoneum; the superior haemorrhoidal artery will have been ligatured in the lower portion of the meso-colon thus tied the lateral ligaments are then tied with the peritoneum covering them and divided; these will include the middle haemorrhoidal artery upon each side; the peritoneum in front, as it is reflected off the front of the rectum, is now divided, thus completing the separation of the peritoneum from the rectum.

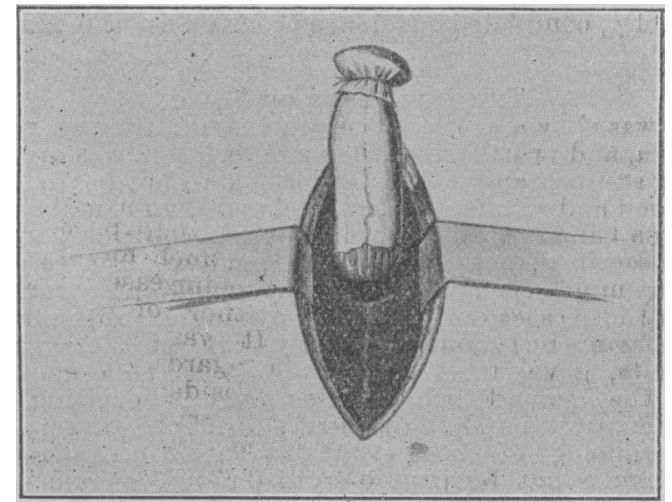

Fig. 9.-Abdominal excision of cancer of the rectum : the lower segments of colon and rectum have been separated from peritoneal attachments : the superior and bath ardale arteries have been tied.

By blunt dissection the bowel can now be cleared right down to the pelvic diaphragm, and, if possible, firmly ligatured below the seat of disease; an assistant should now thoroughly wash out the anal canal and rectum up to the point at which it is ligatured, after which it may be divided from above, below the seat of ligature; the end of the colon is now brought out at the anus and fixed there, after the mucous membrane of the lower segment has been dissected away after the method of Moulonguet, this appears preferable to any attempt at end-to-pnd junction for reasons already given. If it is thought desirable to employ drainage, an

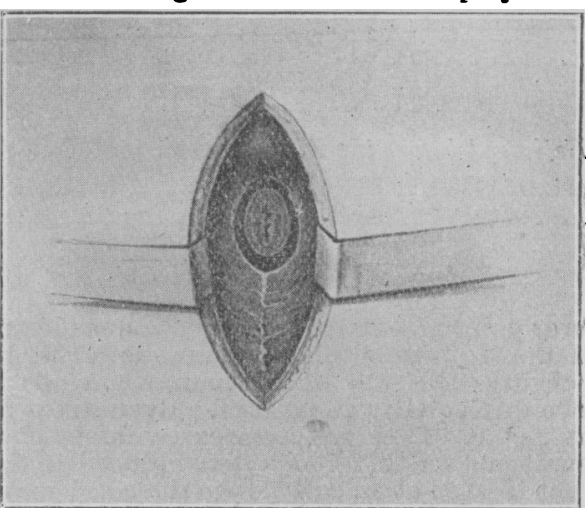

Fig. Io.-Abdominal excision of cancer of the rectum; the rectum has been cut across immediately above the anal the r.

incision for the insertion of a tube of iodoform gauze can be made through the pelvic diaphragm at the apex of the coccyx. I have so far only had personal experience of two cases of the above operation, both were very extensive; there was no difficulty in bringing down the pelvic colon to the anus as above described. The first was a case in which there had been long standing obstruction, and the patient died of gangrenous inflammation of the buttock and lower portion of the abdominal wall, and although the other case only lived 5 weeks, union of the colon to the anus was good and functional result perfect. I believe, however, in less extensive cases good results will be obtained.

Where the meso-colon is too short, the operation must be completed by colotomy as in Fig. 11 .

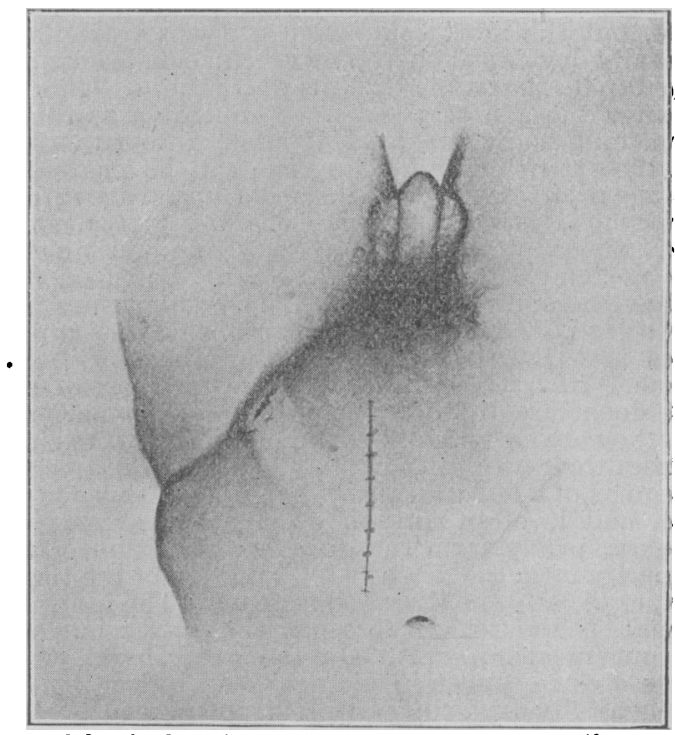

Fig. xx.-Abdominal excision of cancer of the rectum; the upper end of the colon, not having a sufficiently long meso-colon to allow of its being brought down to the anus, is formed into an artificial anus in the groin.

REFERENCE.
'Soc. de Méd. d'Amiens, July, r8go.

"SMaLL-POX HRRe."-Three cases of small-pox in coloured men employed as restaurant waiters were recently detected in Chicago. The restanrant keepers were expostulated with for employing unvaccinated attendants, but protested that they could not enforce vaccination as a condition of employment. In consequence the chief medical inspector was instructed to post. a placard, "Small-pox here," at the antrance of the next restaurant in which a small-pox case is found, and to place a sanitary officer at the door to warn people away until all necessary preventive measures of disinfection, vaccination, etc., had been enforced. 